\title{
Search for MSSM Higgs boson decaying to a pair of tau leptons in CMS
}

\author{
Yiwen Wen ${ }^{* \dagger}$ \\ Deutsches Elektronen-Synchrotron (DE) \\ E-mail: viwen.wendcern.ch
}

The latest results of the search for a neutral Higgs boson decaying into two tau leptons with the full 2016 data are presented. The analysis is performed using the dataset recorded by the CMS experiment at the LHC from pp collisions at centre-of-mass energies of $13 \mathrm{TeV}$ corresponding to an integrated luminosity of $12.9 / \mathrm{fb}$. Results are interpreted within the context of minimal supersymmetric model for a range of signal masses between 90 and $3200 \mathrm{GeV}$. No signal has been found and exclusion limits are presented

The European Physical Society Conference on High Energy Physics

5-12 July

Venice, Italy

*Speaker.

${ }^{\dagger}$ on behalf of CMS collaboration 


\section{Introduction}

The standard model (SM) predicts one neutral scalar Higgs boson. In July 2012, one neutral boson was discovered at the Large Hadron Collider with a mass of $125.09 \pm 0.21$ (stat.) \pm 0.11 (syst) $\mathrm{GeV}[\mathrm{U}, \mathrm{D}]$. Besides the SM, there are a lot of theories beyond the SM that predict additional Higgs bosons. The minimal supersymmetric standard model (MSSM) is one of the simplest extension model of the SM[3], 团]. In the Higgs boson sector of the MSSM, there are two Higgs doublets which lead to five physical Higgs bosons: the charged $H^{ \pm}$pair and three neutral bosons: the light (h) and heavy $(\mathrm{H})$ scaler Higgs bosons and pseudoscalar A.

At tree level, the properties of these particles are described by two free parameters, conventionally chosen as the mass of the pseudoscalar $m_{A}$ and the vacuum expectation values of the two doublets $\tan \beta$. For low and medium $\tan \beta$, gluon-gluon fusion process dominates. While for high $\tan \beta$, associated production with $\mathrm{b}$-jet is the dominant one. The leading order diagrams can be seen in Figure $\mathbb{W}$. With respect to SM Higgs to $\tau \tau$, the branching ratios of $H \rightarrow \tau \tau$ and $A \rightarrow \tau \tau$ are enhanced in high $\tan \beta$ parameter space.
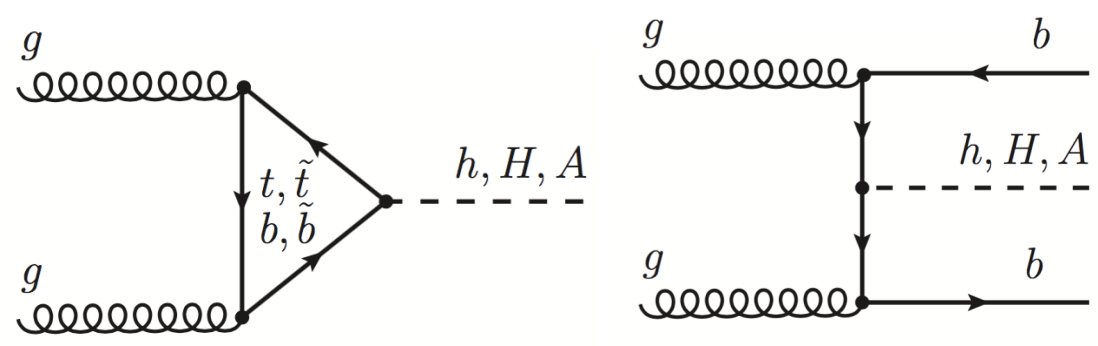

Figure 1: Leading order diagrams of the gluon fusion(left) and b-associated Higgs production(right) in four-flavour scheme.

A search for a MSSM neutral Higgs boson decaying into pair of $\tau$ leptons is performed using Run 2 data collected with the CMS experiment at the LHC during the early 2016 run period with $12.9 \mathrm{fb}^{-1}$ integrated luminosity at the center of mass energy of $13 \mathrm{TeV}$ [可.

\section{2. $\tau$ identification}

The analysis is performed for four final states of the $\tau$ decays, $e \mu, e \tau_{h}, \mu \tau_{h}$ and $\tau_{h} \tau_{h}$, where the hadronic $\tau$ decay is denoted as $\tau_{h}$.

In CMS experiment, hadronic decay $\tau$ is reconstructed by using hadron-plus-strip (HPS) algorithm[ $[$ ] . The algorithm is designed to reconstruct individual decay modes of the $\tau$, taking advantage of the excellent performance of the particle-flow (PF) algorithm[D] to reconstruct individual charged hadrons, neutral hadrons, electrons, photons and muons. There are two stages for HPS algorithm:

- Reconstruction step: Combine charged hadrons and cluster the photon constituents into $\eta-\phi$ strip. Then counting how many charged hadrons and how many strips, a combinatorial approach is taken to correspond to different hadronic $\tau$ decay modes(Figure $\square$ ). 
- Identification step: Discriminators that separate hadronic $\tau$ decays from quark and gluon jets, from electrons and from muons are computed in the second step.
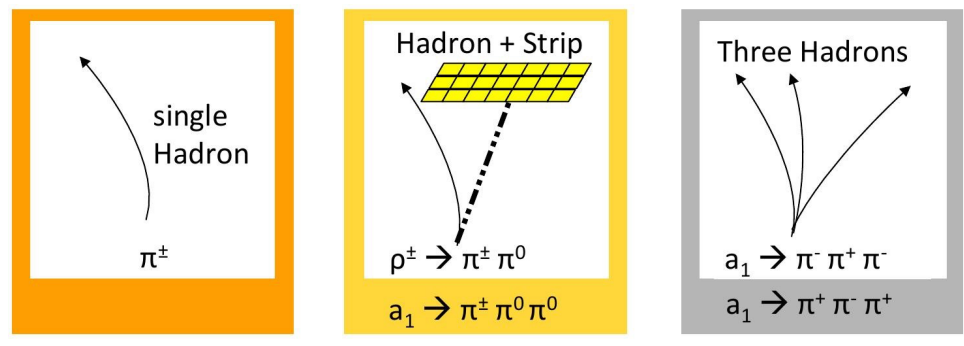

Figure 2: 1-prong(left), 1-prong $+\pi_{0}$ (middle) and 3-prongs(right) decay modes.

3. $H / A \rightarrow \tau \tau$

All particle objects such as muons, electrons, photons, charged and neutral hadrons are reconstructed using PF algorithm by collecting information coming from all the sub-detectors of CMS. Events above the trigger threshold are selected by applying offline $p_{T}$ and $\eta$ cuts within the geometric acceptance in all four channels of the $\tau$ decay. Simulated Monte Carlo (MC) samples are generated for various ranges of signal mass $\left(m_{\phi}=90-3200 \mathrm{GeV}\right)$. Background events for all channels including the Drell-Yan $Z / \gamma^{*}+$ jets, $\mathrm{W}+$ jets, diboson(WW, WZ, ZZ), $t \bar{t}$, and the single top processes are estimated form $\mathrm{MC}$ samples and with data driven corrections applied. QCD multi-jet events is estimated from control regions in data.

To focus on one or the other production mode, the analysis is performed in two different event categories: one requiring at least one b-tagged jet and the other vetoing b-tagged jet. For gluon-gluon production, events with exactly zero b-tagged jet are selected. To target $b$-associated production, selected events have at least one b-tagged jet with $p_{T}>20 \mathrm{GeV}$ and $|\eta|<2.4$. These b-tagged jets are defined as passing the "medium" working point criteria of the combined secondary vertex (CSV) discriminator[[]].

To extract the signal, we use total transverse mass variable as final discriminant:

$$
m_{T}^{\text {tot }}=\sqrt{m_{T}\left(E_{T}^{m i s s}, \tau_{1}^{v i s}\right)^{2}+m_{T}\left(E_{T}^{m i s s}, \tau_{2}^{v i s}\right)^{2}+m_{T}\left(\tau_{1}^{v i s}, \tau_{2}^{v i s}\right)^{2}}
$$

where $m_{T}=\sqrt{2 p_{T}^{1} P_{T}^{2}(1-\cos \Delta \phi)}, E_{T}^{\text {miss }}$ is the missing transverse energy, $\tau_{1}^{v i s}$ is the leading $\tau$ visible decay product, $\tau_{2}^{v i s}$ is the second leading $\tau$ visible decay product and $\Delta \phi$ is the azimuthal angle between different decay products.

This discriminant is then fitted using maximum likelihood fit in all four channels in the no btagged jet and with b-tagged jet categories (Figure B]). Additional control regions are also included in the fit. After fitting the total transverse mass variable, we set model independent upper limits on the cross-section times branching ratios for both gluon-gluon and b-associated Higgs production. Figure $\mathbb{A}$ shows the $95 \%$ confidential level upper limit on $\sigma \cdot B$ for the combination of all four channels in both categories. $m_{h}^{\bmod +}$ [0] and hMSSM[ए0] scenarios are used to set the model 
dependent exclusion limits as shown in Figure 5. 2D likelihood contours for fitting both signal productions are also shown in Figure 6 . These are produced for different values of the Higgs boson mass $m_{\Phi}$ as shown in figures. The scans are made with the constraint that the signal cross-section time branching fraction should be $\geq 0$.
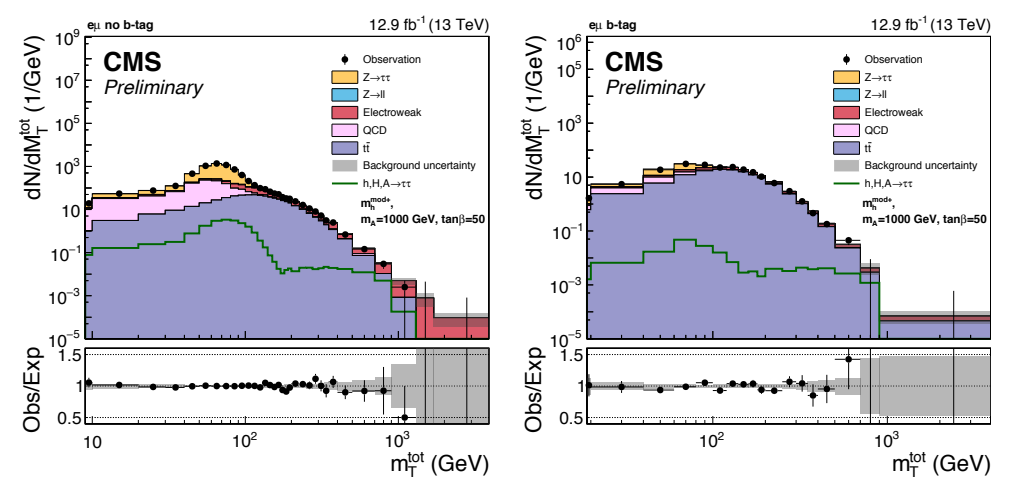

Figure 3: No b-tag (left) and b-tag (right) categories post fit plots of total transverse mass distribution in the $e \mu$ channel.
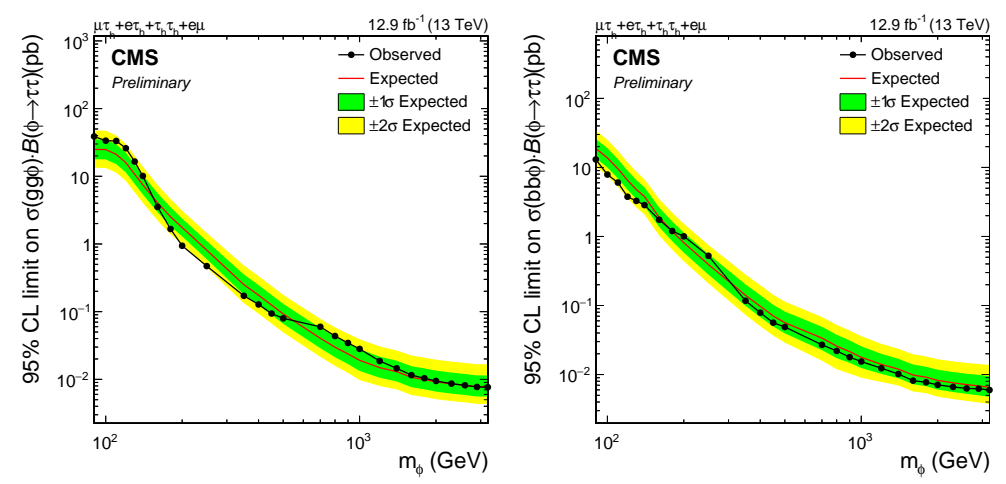

Figure 4: Expected and observed model independent limits on the $\sigma(g g \phi) \cdot B(\phi \rightarrow \tau \tau)$ (left) and $\sigma(b b \phi)$. $B(\phi \rightarrow \tau \tau)$ (right).

\section{Conclusion}

No evidence for excessive signal events has been found for a neutral MSSM Higgs boson decaying into a $\tau \tau$ pair in a dataset corresponding to an integrated luminosity of $12.9 \mathrm{fb}^{-1}$ at 13 $\mathrm{TeV}$ center-of-mass energy using $e \mu, \mu \tau_{h}, e \tau_{h}$ and $\tau_{h} \tau_{h}$ final states. Exclusion limits are set on the production cross section times branching fraction of the gluon-gluon fusion and b-associated production modes. For two MSSM benchmark scenarios, the exclusion limits are set as a function of $m_{A}$ and $\tan \beta$. 2D likelihood contours for both signal productions are also presented. 

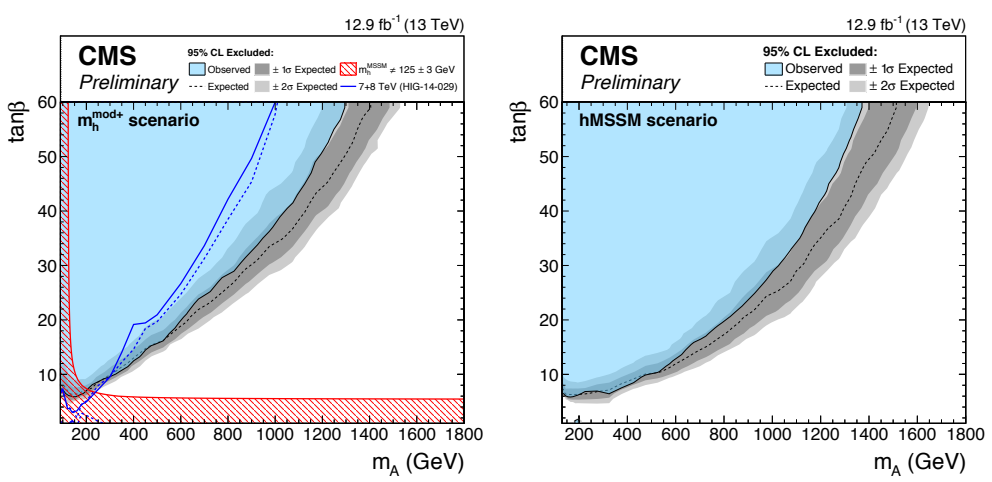

Figure 5: Model dependent limits combining all channels in $m_{A}-\tan \beta$ plane, for $m_{h}^{\text {mod }}+$ scenario (left) and the hMSSM (right). In the left, the blue lines indicate the expected (dashed) and the observed (solid) exclusions obtained from Run 2 CMS search and the red contour indicates the region which does not yield a Higgs boson consistent with a mass of $125 \mathrm{GeV}$ within the theory uncertainties of $\pm 3 \mathrm{GeV}$.

\section{References}

[1] CMS Collaboration, "Observation of a new boson at a mass of $125 \mathrm{GeV}$ with the CMS experiment at the LHC", Phys. Lett B716 (2012) 30-61,

[2] ATLAS Collaboration, "Observation of a new particle in the search for Standard Model Higgs boson with the ATLAS detector at the LHC", Phys. Lett B716 (2012) 1-29,

[3] P. Fayet, "Supergauge invariant extension of the Higgs mechanism and a model for the electron and its neutrino", Nucl. Phys. B 90(1975) 104,

[4] P. Fayet, "Spontaneously broken supersymmetric theories of weak, electromagnetic and strong interations", Phys. Lett. B 69(1977) 489,

[5] CMS Collaboration, "Search for a neutral MSSM Higgs boson decaying into $\tau \tau$ with $12.9 \mathrm{fb}^{-1}$ at $\sqrt{s}=13$ TeV", CMS-PAS-HIG-16-037, CERN(2016),

[6] CMS Collaboration, "Performance of reconstruction and identification of tau leptons in their decays to hadrons and tau neutrino in LHC Run-2", CMS-PAS-TAU-16-002, CERN(2016),

[7] CMS Collaboration, "Particle-Flow event reconstruction in CMS and performance for jets, taus and missing $E_{T}$ ", CMS-PAS-PFT-09-001, CERN(2009),

[8] CMS Collaboration, "Identification of b and c jets in the CMS experiment at $13 \mathrm{TeV}$ ", CMS-PAS-BTV-16-002, CERN(2016),

[9] M. Carena et al., "MSSM Higgs boson searches at the LHC: benchmark scenarios after the discovery of a Higgs-like particle", Eur. Phys. J C73(2013), no.9, 2552,

[10] L. Maiani, A. D. Polosa, and V. Riquer, "Bounds to the Higgs sector masses in minimal supersymmetric from LHC Data", Phys. Lett. B724(2013) 274-277. 


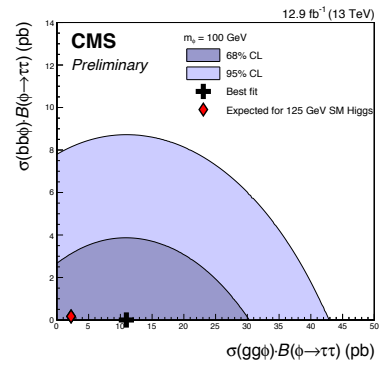

(a) $m_{\phi}=100 \mathrm{GeV}$

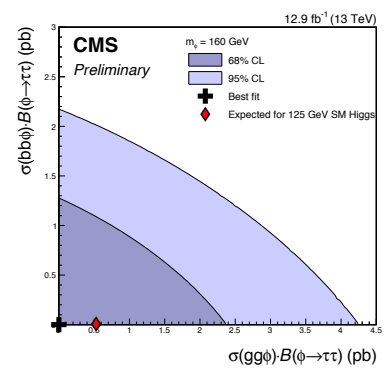

(d) $m_{\phi}=160 \mathrm{GeV}$

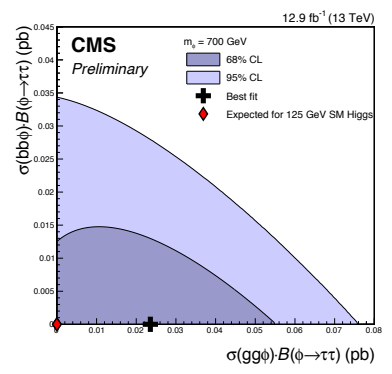

(g) $m_{\phi}=700 \mathrm{GeV}$

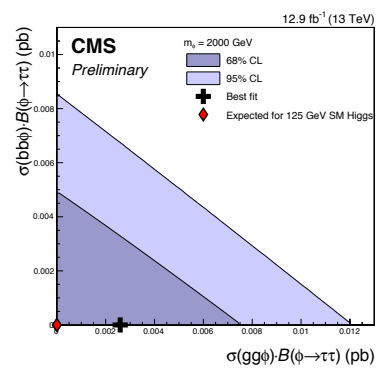

(j) $m_{\phi}=2000 \mathrm{GeV}$

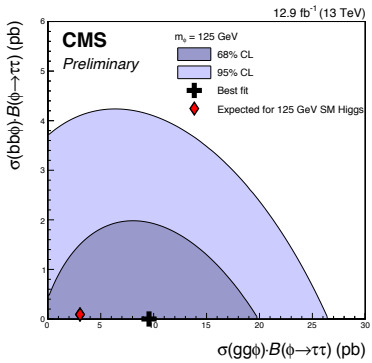

(b) $m_{\phi}=125 \mathrm{GeV}$

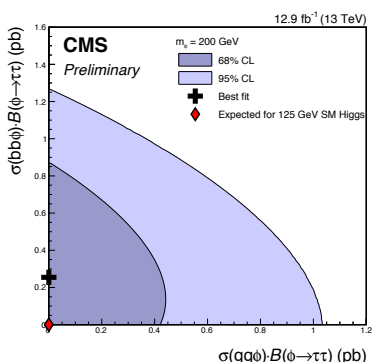

(e) $m_{\phi}=200 \mathrm{GeV}$

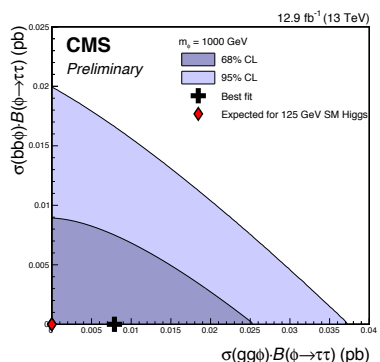

(h) $m_{\phi}=1000 \mathrm{GeV}$

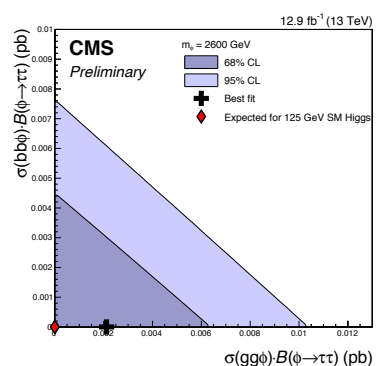

(k) $m_{\phi}=2600 \mathrm{GeV}$

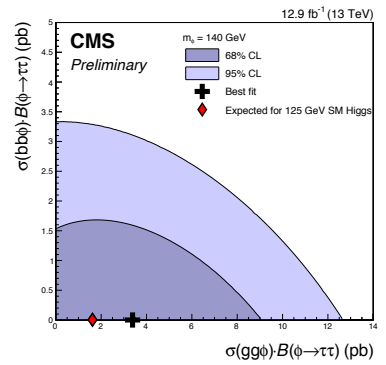

(c) $m_{\phi}=140 \mathrm{GeV}$

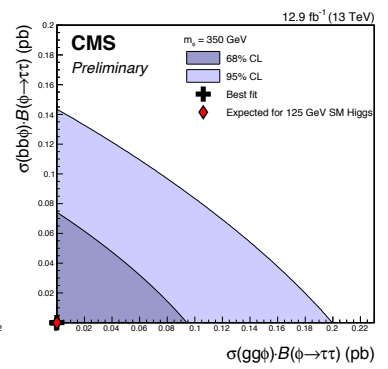

(f) $m_{\phi}=350 \mathrm{GeV}$

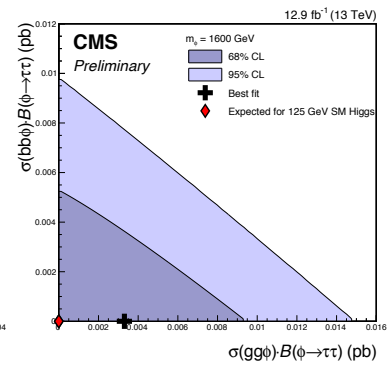

(i) $m_{\phi}=1600 \mathrm{GeV}$

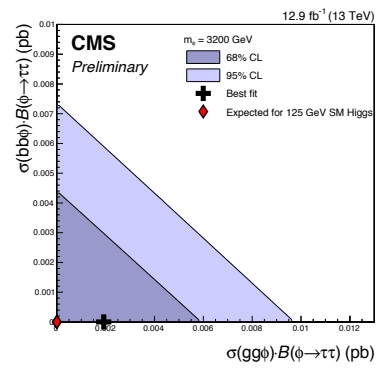

(l) $m_{\phi}=3200 \mathrm{GeV}$

Figure 6: $2 \mathrm{D}$ likelihood scan of cross-section times branching ratio for $g g \phi$ vs. $b b \phi$ production processes, for selected Higgs boson masses between $100 \mathrm{GeV}$ and $3200 \mathrm{GeV}$. The best fit point (black cross) and the 1 and 2 sigma contours are shown for the observed data. Also shown is the best fit value for an Asimov dataset containing background plus the SM Higgs with mass $125 \mathrm{GeV}$ (red diamond). 